\title{
Accurate determination of screw position in treating fifth metatarsal base fractures to shorten radiation exposure time
}

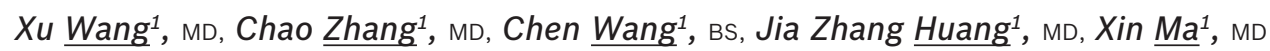

INTRODUCTION Anatomical markers can help to guide lag screw placement during surgery for internal fixation of fifth metatarsal base fractures. This study aimed to identify the optimal anatomical markers and thus reduce radiation exposure. METHODS A total of 50 patients in Huashan Hospital, Shanghai, China, who underwent oblique foot radiography in the lateral position were randomly selected. The angles between the fifth metatarsal axis and cuboid articular surface were measured to determine the optimal lag screw placement relative to anatomical markers.

RESULTS The line connecting the styloid process of the fifth metatarsal base with the second metatarsophalangeal (MTP) joint intersected with the fifth metatarsal base fracture line at an angle of $86.85^{\circ} \pm 5.44^{\circ}$. The line connecting the fifth metatarsal base styloid with the third and fourth MTP joints intersected with the fracture line at angles of $93.28^{\circ} \pm 5.24^{\circ}$ and $100.95^{\circ} \pm 5.00^{\circ}$, respectively. The proximal articular surface of the fifth metatarsal base intersected with the line connecting the styloid process of the fifth metatarsal base with the second, third and fourth MTP joints at angles of $24.02^{\circ} \pm 4.77^{\circ}, 30.79^{\circ} \pm 4.53^{\circ}$ and $38.08^{\circ} \pm 4.54^{\circ}$, respectively.

CONCLUSION The fifth metatarsal base styloid and third MTP joint can be used as anatomical markers for lag screw placement in fractures involving the fifth tarsometatarsal joint. The connection line, which is normally perpendicular to the fracture line, provides sufficient mechanical stability to facilitate accurate screw placement. The use of these anatomical markers could help to reduce unnecessary radiation exposure for patients and medical staff.

Keywords: fifth metatarsal fracture, internal fixation, radiation

\section{INTRODUCTION}

Fracture of the fifth metatarsal base is a common injury that accounts for $45 \%-70 \%$ of all metatarsal fractures. ${ }^{(1)}$ When the foot is in plantar flexion and ankle inversion, the peroneus brevis muscle or plantar fascia contracts strongly, resulting in fifth metatarsal styloid avulsion. (2) If the bone fragment is not displaced or only mildly displaced, the injury can be treated by wearing hard-soled shoes or using cast fixation for a few weeks. However, if the bone is displaced more than $3-5 \mathrm{~mm}$ or the rotational displacement is greater than $10^{\circ}$, surgery is required..$^{(3,4)}$ The choice of surgical method and fixation material varies. Commonly used fixation materials include Kirschner wires, tension bands, cancellous bone screws and intramedullary screws, while common surgical methods include fixation with 4.5-mm ankle screws and transplantation of cortex-cancellous bone after the removal of sclerotic bone from the medullary cavity. These methods have high success rates. ${ }^{(5)}$ The use of lag screws provides an additional mechanical advantage, as they are stronger than single screws. ${ }^{(6)}$

Due to the very small fragments associated with proximal fractures, it is rare to have more than one screw placement opportunity. Thus, almost all operations are performed using a C-arm X-ray image intensifier to ensure correct screw positioning. ${ }^{(6,7)}$ However, this exposes both the medical staff and patient to radiation. A recent survey revealed that more than $75 \%$ of medical staff underestimate the potential risk of exposure to radiation. ${ }^{(8)}$ Between 1980 and 2006, radiography-assisted surgery increased by $47 \%{ }^{(9)}$ Despite the low single doses that are routinely used, the accumulated radiation dose was reported to have increased by $727 \%$. $^{(9)}$ In a survey conducted by Fazel et al, more than 655,000 (69\%) of 950,000 adult patients seeking medical treatment were exposed to a radiation procedure within the three-year study period. ${ }^{(10)}$ Furthermore, the cancer prevalence rate is five times greater among orthopaedic surgeons as compared to other medical staff. ${ }^{(10,11)}$

As orthopaedic surgeons are familiar with the anatomical characteristics of the musculoskeletal system, using anatomical markers to guide internal fixation would help to make surgical procedures safer (by reducing radiation exposure) and more convenient. The centre of the metatarsophalangeal (MTP) joints is the most commonly used anatomical marker in foot surgeries to guide Kirschner wire placement; intraoperatively, passive movement can be applied to determine the centre of the MTP joints. The present study investigated which MTP joint is optimal for use as an anatomical landmark in cases of intra-articular fifth metatarsal fracture (Type II) surgeries. This could help to guide the accurate positioning of internal fixation screws and reduce radiation exposure for patients and medical staff.

\section{METHODS}

A total of 50 patients who underwent foot radiography at the Huashan Hospital of Fudan University, Shanghai, China, between 2010 and 2011 were selected for inclusion in the present study. All 

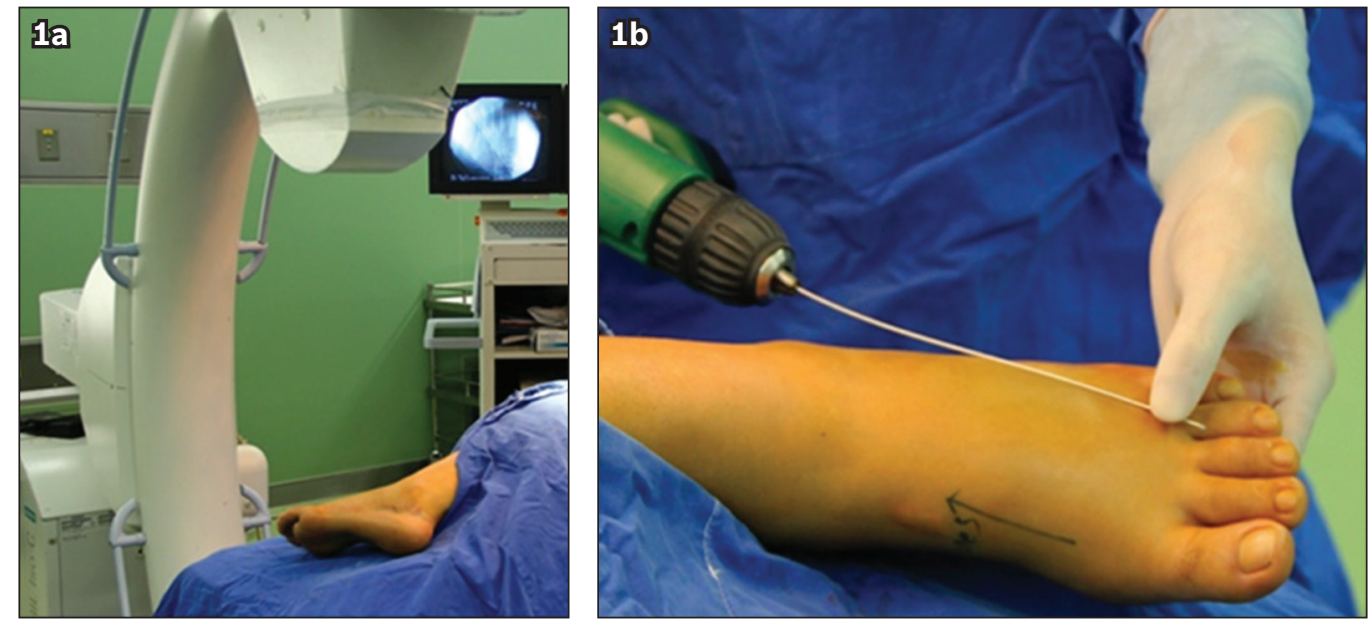

Fig. 1 Photographs show (a) the position of the patient intraoperatively; and (b) the direction of screw placement.
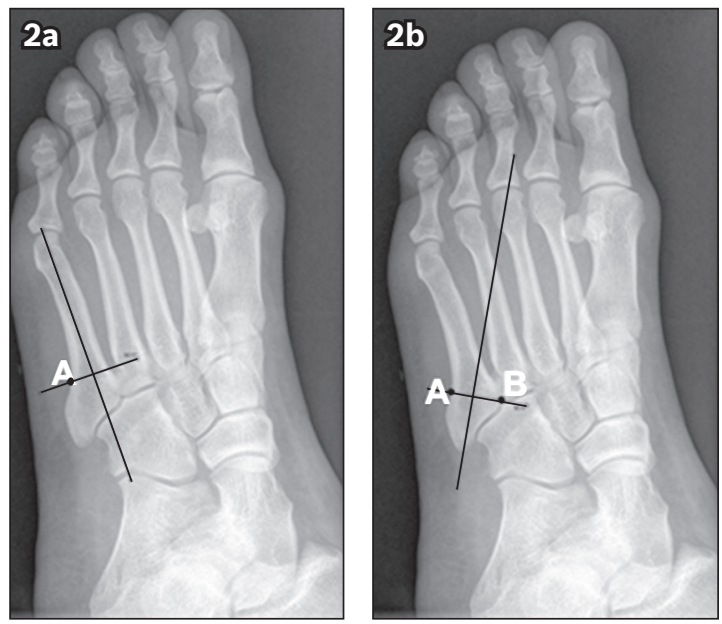

Fig. 2 Radiographs show (a) Point A, the intersection point of the latera cortex of the fifth metatarsal and a perpendicular line of the fifth metatarsal axis; and (b) Point $B$, the nearest point of the articular surface between the fourth and fifth metatarsal base. The proximal part from Line $A B$ is the most common region for bone fractures of the fifth metatarsal base.

patients were confirmed to be free of mid- and forefoot deformities before they were enrolled in the study. Stewart reported that true Jones fractures were transverse fractures at the junction of the diaphysis and metaphysis, without extension to the fourth and fifth intermetatarsal articulations. ${ }^{(12)}$ Thus, cases of genuine Jones fractures were excluded because the main focus of the study was intra-articular fifth metatarsal fractures.

The patients were positioned in a simulated lateral surgical position prior to oblique foot radiography. Pelvic fixation was used to prevent the body from shaking. The contralateral lower limb was flexed between $60^{\circ}$ and $70^{\circ}$, and a padded cushion was placed between the patient's legs, with the injured limb placed on the cushion. The hip joint on the injured side was placed in a neutral position, the knee was flexed $15^{\circ}$, and the ankle was in slight plantar flexion and pronation, perpendicular to the ground. The calcaneocuboid joint was used to mark the centre that was used for oblique foot radiography (Fig. 1).

The metatarsal axis was determined according to the method described by Shima et al; ${ }^{(13)}$ that is, the line connecting the midpoint of the proximal articular surface and centre of the distal articular surface was taken to provide the most accurate axis. A second line, perpendicular to the fifth metatarsal axis, was determined on the oblique view. The line begins at the most distal point of the articular surface between the fourth and fifth metatarsal base, and intersects with the lateral cortex of the fifth metatarsal at Point A (Fig. 2). A third line was drawn to connect Point A to the medial articular surface of the fifth tarsometatarsal (TMT) joint (i.e. Point B). Line $A B$ indicated the extent of the fifth metatarsal base protrusion and roughly corresponded to the tuberosity avulsion fracture line with articular involvement (Fig. 2). The styloid tip of the fifth metatarsal base was determined as Point C (Fig. 3). The midpoint of the articular surfaces of the fourth, third and second MTP joints, respectively, are shown as Points D, E and F in Fig. 3. Lines CD, CE, and CF represent the directions of guide wires.

The angles $\mathrm{d} 2$, e 2 and $\mathrm{f} 2$ were formed by the intersections of the proximal bevel of the fifth metatarsal (i.e. Line BC) with $\mathrm{CD}, \mathrm{CE}$ and $\mathrm{CF}$, respectively (Fig. 3), while the angles $\mathrm{d} 1$, e1 and $\mathrm{f} 1$ were formed by the intersections of $\mathrm{AB}$ with $\mathrm{CD}, \mathrm{CE}$ and $\mathrm{CF}$, respectively (Fig. 4). Each of the angles was measured by two radiologists and two orthopaedic surgeons using computerassisted design software. Measurements were repeated and mean values were calculated.

\section{RESULTS}

The line connecting the fifth metatarsal base styloid with the second MTP joint intersected with the fifth metatarsal base fracture line at a mean angle of $86.85^{\circ} \pm 5.44^{\circ}$ (Table I). The line connecting the fifth metatarsal base styloid with the third and fourth MTP joints intersected with the fracture line at mean angles of $93.28^{\circ} \pm 5.24^{\circ}$ and $100.95^{\circ} \pm 5.00^{\circ}$, respectively. The proximal articular surface of the fifth metatarsal base intersected with the line connecting the fifth metatarsal base styloid with the second, third and fourth MTP joints at mean angles of $24.02^{\circ} \pm$ $4.77^{\circ}, 30.79^{\circ} \pm 4.53^{\circ}$, and $38.08^{\circ} \pm 4.54^{\circ}$, respectively.

\section{DISCUSSION}

The present study aimed to identify the anatomical markers that can be used to define the optimal lag screw location during surgery 

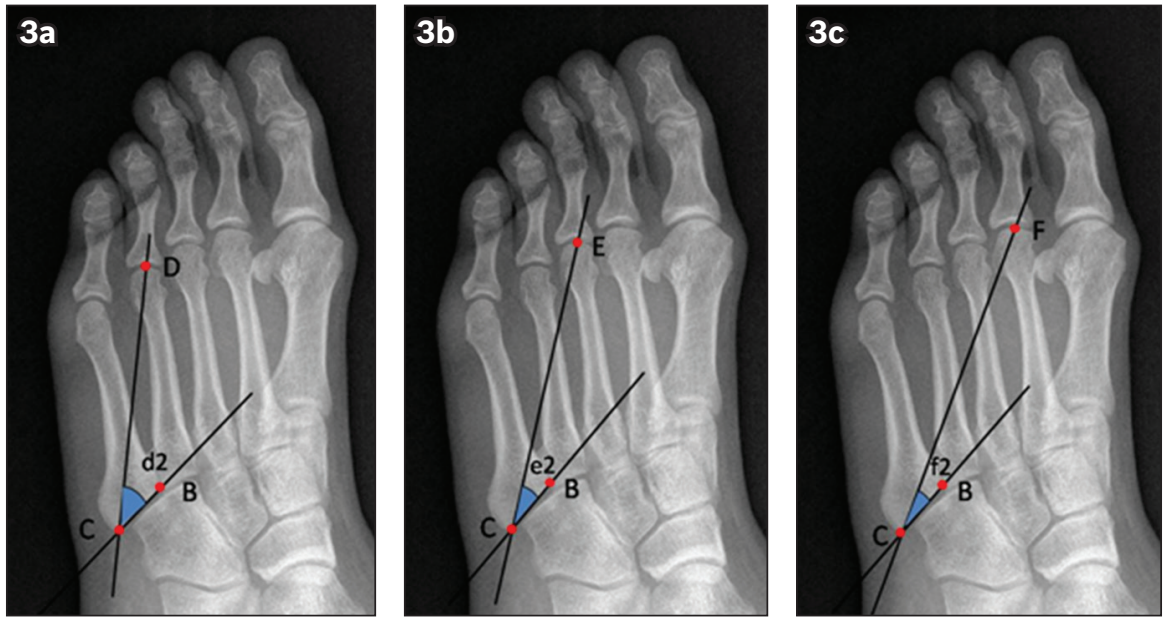

Fig. 3 Radiographs show the determination of the angles between the proximal articular surface of the fifth metatarsal and the possible placement of the screw. (a) Line BC marks the proximal articular surface of the fifth metatarsal and Line $C D$ is formed by joining the nearest point of the fifth metatarsal base (Point $\mathrm{C}$ ) and the midpoint of the fourth metatarsal joint (Point $\mathrm{D}$ ). The resulting angle $\mathrm{d} 2$ is too large. (b) Line CE is formed by joining Point $\mathrm{C}$ and the midpoint of the third metatarsal joint (Point E). The resulting angle e2 is ideal. (c) Line CF is formed by joining Point $C$ and the midpoint of the second metatarsal joint (Point F). The resulting angle $\mathrm{f} 2$ is too small.
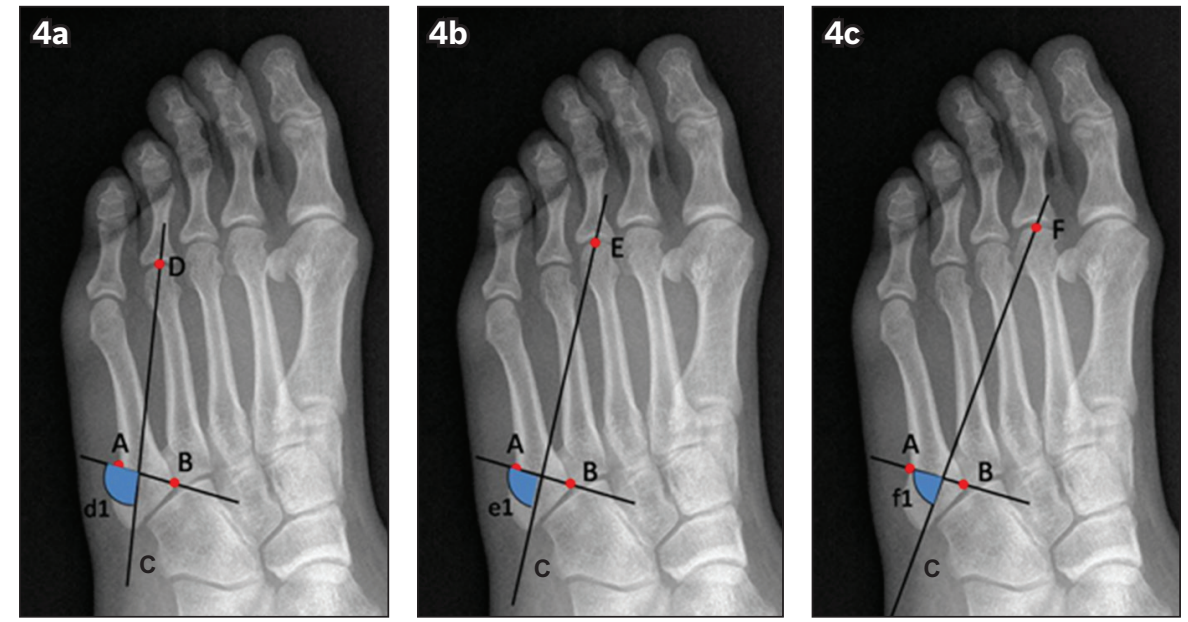

Fig. 4 Radiographs show the determination of the angles between the proximal articular surface of the fifth metatarsal and the possible placement of the screw, with the angles (a) d1 between Lines $A B$ and CD; (b) e1 between Lines AB and CE; and (c) f1 between Lines AB and CF.

Table I. Angle measurements.

\begin{tabular}{lcc}
\hline Angle $\left(^{\circ}\right)$ & Mean \pm SD & Range \\
\hline d2 & $38.08^{\circ} \pm 4.54^{\circ}$ & $28.94^{\circ}-49.65^{\circ}$ \\
e2 & $30.79^{\circ} \pm 4.53^{\circ}$ & $22.20^{\circ}-42.43^{\circ}$ \\
f2 & $24.02^{\circ} \pm 4.77^{\circ}$ & $15.47^{\circ}-35.75^{\circ}$ \\
d1 & $100.95^{\circ} \pm 5.00^{\circ}$ & $90.73^{\circ}-111.54^{\circ}$ \\
e1 & $93.28^{\circ} \pm 5.24^{\circ}$ & $83.19^{\circ}-104.28^{\circ}$ \\
f1 & $86.85^{\circ} \pm 5.44^{\circ}$ & $75.97^{\circ}-99.20^{\circ}$ \\
\hline
\end{tabular}

SD: standard deviation

for internal fixation of fifth metatarsal base fractures. The use of these anatomical markers can help to reduce radiation exposure among patients and medical staff. We measured various angles for lag screw placement in order to locate the ideal direction. In accordance with the AO (Association for the Study of Internal Fixation) principles of internal fixation, the tension screw has the greatest strength when it is vertical to the fracture line. Hence, the angle arising from the third MTP joint was considered the most optimal, as it was close to $90^{\circ}\left(93.28^{\circ} \pm 5.24^{\circ}\right)$. The results showed that the fifth metatarsal base styloid and the third MTP joint can be used as anatomical markers for screw placement in fractures involving the fifth TMT joint. The connection line, which is normally perpendicular to the fracture line, provides quick and accurate screw placement.

The pathological mechanism, diagnosis and treatment of fifth metatarsal base fractures are controversial. ${ }^{(6)}$ Generally, the articular surfaces of the proximal fourth and fifth metatarsal bases have been used to distinguish between extra-articular (Type I) and intra-articular (Type II) fifth metatarsal fractures. ${ }^{(14)}$ In the present study, the metatarsal axis was determined according to the method described by Shima et al, in which the line connecting the midpoint of the proximal articular surface and centre of the distal articular surface provided the most accurate metatarsal axis. ${ }^{(13)} \mathrm{A}$ second line perpendicular to the fifth metatarsal axis was determined on the oblique view. This line, which begins at the most distal point of the joint along the fourth and fifth metatarsal base, intersects with the lateral cortex of the fifth metatarsal at Point A (Fig. 2). A third line was then drawn to connect Point A to the medial articular surface of the fifth TMT joint (i.e. Point B); Line $A B$ indicated the extent of the fifth metatarsal base protrusion 

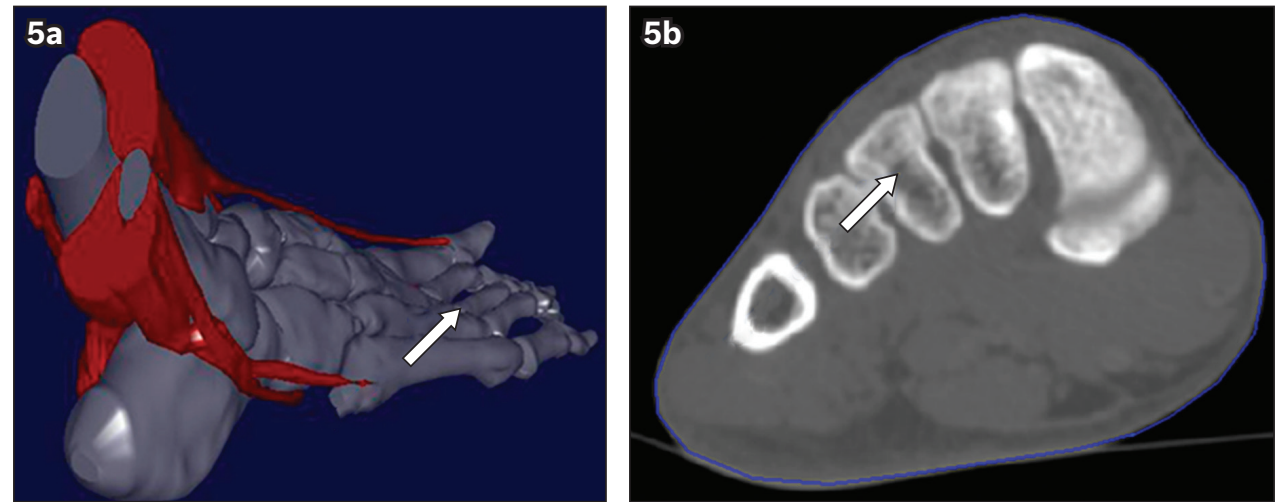

Fig. 5 (a) 3D-reconstructed and (b) coronal CT images show the direction of screw placement in the midpoint of the third metatarsal joint (arrows).
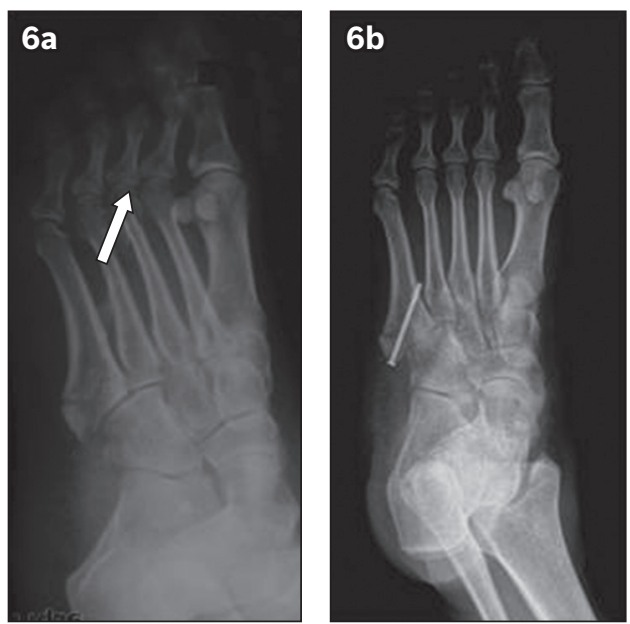

Fig. 6 Radiographs show the (a) hypothetical screw direction, with the screw pointing toward the midpoint of the metatarsal joint (arrow) and (b) postoperative screw direction.

and simulated the tuberosity avulsion fracture line with articular involvement (Fig. 2).

The basic treatment principle is to keep the screw perpendicular to the fracture line to achieve the most secure fixation. Mechanical experiments have shown that screws positioned vertical to the fracture line and bicortical fixation provide maximum strength. ${ }^{(4)}$ Screw fixation in the proximal articular surface involves the fifth TMT joint, whereas distal fixation involves the third, fourth or fifth metatarsal proximal joint. In our study, the direction perpendicular to the fracture line provided maximal biomechanical strength, at an angle of $93.28^{\circ} \pm 5.24^{\circ}$ to the third MTP joint. The line projected to the second MTP joint also intersected with the fracture line at a right angle. However, we do not recommend it as a guiding marker, because its angle with the articular surface of the fifth TMT joint (i.e. f2) is too small if the centre of the second MTP joint is used $\left(24.02^{\circ}\right.$ on average). This could cause the screw to enter the fifth TMT joint or even the proximal articular surface between the fourth and fifth metatarsals, damaging the articular surface. The line connecting the fifth metatarsal base point and fourth MTP joint formed an angle of $100.95^{\circ} \pm 5.00^{\circ}$ with the fracture line; this would reduce mechanical strength and hamper screw fixation in the fifth metatarsal lateral cortex. In addition, using the second or fourth MTP joint could result in medial or lateral deviation.
Due to the transverse arch of the foot, the fifth metatarsal base is closer to the plantar side of the foot than to the third MTP joint. ${ }^{(15,16)}$ In our opinion, screws fixed from the styloid of the fifth metatarsal base pointing obliquely to the third MTP joint should be aligned in a sagittal position to avoid the risk of piercing through the plantar or dorsal side (Fig. 5). This surgical procedure, which is supplemented by the use of brief $X$-ray radiation for verification, was easy to perform, maximised lag screw mechanical strength, and avoided repeated use of Kirschner wires and thus the associated risk of fragmentation of the proximal fractures (Fig. 6). Although the use of the C-arm X-ray is still needed during surgery to ensure the correct direction and position of the screw, the radiation dose is substantially lower if anatomical markers, instead of the C-arm X-ray, are used to determine the position of the lag screw.

In conclusion, the present study showed that patients with fifth metatarsal base fractures should undergo surgery in the lateral position, with the injured limb hip joint placed in a neutral position, the knee flexed to an angle of approximately $15^{\circ}$ and the ankle positioned in slight plantar flexion. The connection between the styloid tip of the fifth metatarsal base and third MTP joint can be used to determine the position of the leading guide wire for bicortical fixation screws. The use of this anatomical marker as a reference point provides a reliable method for facilitating the surgical repair of fifth metatarsal base fractures and minimises the radiation exposure among patients and medical staff. Prospective studies can be conducted to validate this method.

\section{REFERENCES}

1. Habbu RA, Marsh RS, Anderson JG, Bohay DR. Closed intramedullary screw fixation for nonunion of fifth metatarsal Jones fracture. Foot Ankle Int 2011; 32:603-8.

2. Thomas JL, Davis BC. Three-wire fixation technique for displaced fifth metatarsal base fractures. J Foot Ankle Surg 2011; 50:776-9.

3. Rettig AC, Shelbourne KD, Wilckens J. The surgical treatment of symptomatic nonunions of the proximal (metaphyseal) fifth metatarsal in athletes. Am J Sports Med 1992; 20:50-4

4. Husain ZS, DeFronzo DJ. Relative stability of tension band versus twocortex screw fixation for treating fifth metatarsal base avulsion fractures. J Foot Ankle Surg 2000; 39:89-95.

5. Glasgow MT, Naranja RJ Jr, Glasgow SG, Torg JS. Analysis of failed surgical management of fractures of the base of the fifth metatarsal distal to the tuberosity: the Jones fracture. Foot Ankle Int 1996; 17:449-57.

6. Moshirfar A, Campbell JT, Molloy S, Jasper LE, Belkoff SM. Fifth metatarsal tuberosity fracture fixation: a biomechanical study. Foot Ankle Int 2003; 24:630-3. 
7. Hatch RL, Alsobrook JA, Clugston JR. Diagnosis and management of metatarsal fractures. Am Fam Physician 2007; 76:817-26.

8. Keijzers GB, Britton CJ. Doctors' knowledge of patient radiation exposure from diagnostic imaging requested in the emergency department. Med Aust 2010; 193:450-3.

9. Giordano BD, Grauer JN, Miller CP, Morgan TL, Rechtine GR 2nd Radiation exposure issues in orthopaedics. J Bone Joint Surg Am 2011; 93:e69(1-10)

10. Fazel R, Krumholz HM, Wang Y, et al. Exposure to low-dose ionizing radiation from medical imaging procedures. N Engl J Med 2009; 361:849-57.

11. Mastrangelo G, Fedeli U, Fadda E, et al. Increased cancer risk among surgeons in an orthopaedic hospital. Occup Med (Lond) 2005; 55:498-500.
12. Stewart IM. Jones's fracture: fracture of base of fifth metatarsal. Clin Orthop 1960; 16:190-8

13. Shima H, Okuda R, Yasuda T, et al. Radiographic measurements in patients with hallux valgus before and after proximal crescentic osteotomy. J Bone Joint Surg Am 2009; 91:1369-76.

14. Hartog J. Wither Dutch corporatism? Two decades of employment policies and welfare reforms. Scott J Polit Econ 1999; 46:458-86.

15. Kanatli $U$, Yetkin $\mathrm{H}$, Bolukbasi S. Evaluation of the transverse metatarsal arch of the foot with gait analysis. Arch Orthop Trauma Surg 2003; 123:148-50.

16. Sammarco VJ. The talonavicular and calcaneocuboid joints: anatomy, biomechanics, and clinical management of the transverse tarsal joint. Foot Ankle Clin 2004; 9:127-45. 